PROCEEDINGS OF THE

AMERICAN MATHEMATICAL SOCIETY

Volume 129, Number 7, Pages 2127-2133

S 0002-9939(00)05776-

Article electronically published on November 22, 2000

\title{
MONOTONIC APPROACH TO CENTRAL LIMITS
}

\author{
JONATHAN M. KANE \\ (Communicated by Wei-Yin Loh)
}

\begin{abstract}
The approach to limits guaranteed by the Central Limit Theorem appears to be monotonic in many cases. A variety of empirical examples are discussed. Proofs are given for some special cases of the binomial, gamma, and Poisson distributions.
\end{abstract}

\section{Monotonicity Phenomenon}

Let $F$ be a distribution with mean $\mu$ and standard deviation $\sigma$. Let $X_{1}, X_{2}$, $X_{3}, \ldots$ be a sequence of independent random variables all with distribution $F$. The Central Limit Theorem states that as $n$ approaches infinity, the tail probability

$$
P\left(\sum_{j=1}^{n} X_{j} \geq n \mu+\sqrt{n} m \sigma\right)
$$

will approach $1-\Theta(m)$ where $\Theta$ is the cumulative distribution function of the standard normal distribution. Estimating how closely $1-\Theta(m)$ approximates the tail probability is often important yet difficult. In many cases, though, the approach to the central limit is monotonic, which makes the problem of evaluating the accuracy of the estimates much easier.

For discrete distributions, $F$, that take on only integer values, the values of (1.1) rise and fall as $n \mu+\sqrt{n} m \sigma$ increases to and passes integer values. So, when $F$ is discrete, (1.1) will be discussed only for those $n$ where $n \mu+\sqrt{n} m \sigma$ is integral. The following notation will be used to represent probability distributions. $\operatorname{BIN}(N, p)$ will refer to the binomial distribution with $N$ trials and probability of success $p$. In this case $q$ will be used to represent $1-p \cdot \operatorname{GAM}(\alpha)$ will refer to the gamma distribution with density $\frac{1}{\Gamma(\alpha)} x^{\alpha-1} e^{-x}$ for $x>0$. POI $(\lambda)$ will refer to the Poisson distribution with mean $\lambda$.

Determining conditions under which the monotonicity of (1.1) holds is the subject of this paper. In Section 2 three theorems are presented. In Section 3 numerical evidence of monotonicity is discussed.

\section{AnAlytic Results}

This section presents three results concerning the monotonicity of (1.1) in the special case where $m=0$.

Received by the editors May 24, 1999 and, in revised form, November 15, 1999.

2000 Mathematics Subject Classification. Primary 62E20, 62F12, 62F05.

Key words and phrases. Central limit, binomial distribution, gamma distribution.

(C)2000 American Mathematical Society 
Theorem 2.1. Let $p$ be any rational number with $0<p<1$, and let $N$ be any positive integer where $N p$ is an integer. Let $X_{1}, X_{2}, X_{3}, \ldots$ be a sequence of independent random variables all with distribution $\operatorname{BIN}(N, p)$. Then, $P\left[\sum_{j=1}^{n} X_{j} \geq n(N p)\right]$ is a strictly decreasing function of $n$.

Theorem 2.2. Let $X_{1}, X_{2}, X_{3}, \ldots$ be a sequence of independent random variables all with distribution $\operatorname{GAM}(\alpha)$. Then, $P\left[\sum_{j=1}^{n} X_{j} \geq n \alpha\right]$ is a strictly increasing function of $n$.

Theorem 2.3. Let $\lambda$ be any positive integer and let $X_{1}, X_{2}, X_{3}, \ldots$ be a sequence of independent random variables all with distribution $\mathrm{POI}(\lambda)$. Then, $P\left[\sum_{j=1}^{n} X_{j} \geq n \lambda\right]$ is a strictly decreasing function of $n$.

Theorem 2.1] was proved by Jogdeo and Samuels [1968] using different techniques. The technique used in this paper generalizes to give the results of Theorems 2.2 and 2.3 and may more easily generalize to the cases where $m$ in (1.1) is not 0 .

The following lemma is the key to the proofs of Theorems [2.1] and [2.2.

Lemma. For $0 \leq a<b<c \leq \infty$ let $K(x)$ be a continuously differentiable function on $(a, c)$, increasing on the interval $(a, b)$, decreasing on the interval $(b, c)$, reaching a maximum of $M$ at $b$, and approaching 0 at a and $c$ fast enough to ensure

$$
\lim _{x \rightarrow a^{+}} K(x)^{T} \ln (x)=\lim _{x \rightarrow c^{-}} K(x)^{T} \ln (x)=0
$$

for some $T>0$. Let $G(y)$ be the inverse of $K(x)$ on $(a, b)$ and let $H(y)$ be the inverse of $K(x)$ on $(b, c)$. Suppose that, for any $r>0, G(y) H(y)^{r}$ is decreasing at $y=y_{0}$ only if it is decreasing for all $y$ in $\left(y_{0}, M\right)$. Then the function

$$
f(t)=\frac{\int_{a}^{b} K(x)^{t} \frac{d x}{x}}{\int_{a}^{c} K(x)^{t} \frac{d x}{x}}
$$

is a strictly decreasing function of $t$ for $t>T$.

Proof of the Lemma. The derivative of $\ln [f(t)]$ is

$$
\frac{\int_{a}^{b} K(x)^{t} \ln (K(x)) \frac{d x}{x}}{\int_{a}^{b} K(x)^{t} \frac{d x}{x}}-\frac{\int_{a}^{c} K(x)^{t} \ln (K(x)) \frac{d x}{x}}{\int_{a}^{c} K(x)^{t} \frac{d x}{x}}
$$

which is negative if and only if

$$
\frac{\int_{a}^{b} K(x)^{t} \ln (K(x)) \frac{d x}{x}}{\int_{a}^{b} K(x)^{t} \frac{d x}{x}}-\frac{\int_{b}^{c} K(x)^{t} \ln (K(x)) \frac{d x}{x}}{\int_{b}^{c} K(x)^{t} \frac{d x}{x}}
$$

is negative. Making the change of variables $x=G(y)$ in the integrals of the first fraction and $x=H(y)$ in the integrals of the second fraction gives

$$
\frac{\int_{0}^{M} y^{t} \ln (y) \frac{G^{\prime}(y) d y}{G(y)}}{\int_{0}^{M} y^{t} \frac{G^{\prime}(y) d y}{G(y)}}-\frac{\int_{0}^{M} y^{t} \ln (y) \frac{H^{\prime}(y) d y}{H(y)}}{\int_{0}^{M} y^{t} \frac{H^{\prime}(y) d y}{H(y)}}
$$

Let $I=\int_{0}^{M} y^{t} \frac{G^{\prime}(y) d y}{G(y)}$ and $r=-I / \int_{0}^{M} y^{t} \frac{H^{\prime}(y) d y}{H(y)}$. Then the lemma would follow if the following were shown to be negative:

$$
\frac{\int_{0}^{M} y^{t} \ln (y) \frac{G^{\prime}(y) d y}{G(y)}+r \int_{0}^{M} y^{t} \ln (y) \frac{H^{\prime}(y) d y}{H(y)}}{\int_{0}^{M} y^{t} \frac{G^{\prime}(y) d y}{G(y)}}=\frac{\int_{0}^{M} y^{t} \ln (y)\left[\frac{G^{\prime}(y)}{G(y)}+r \frac{H^{\prime}(y)}{H(y)}\right] d y}{I}
$$


Integrating by parts with $u=\ln (y)$ and $d v=y^{t}\left[\frac{G^{\prime}}{G}+r \frac{H^{\prime}}{H}\right] d y$ gives

$$
\frac{1}{I}\left\{\left.\ln (y) \int_{0}^{y} s^{t}\left[\frac{G^{\prime}(s)}{G(s)}+r \frac{H^{\prime}(s)}{H(s)}\right] d s\right|_{y=0} ^{y=M}-\int_{0}^{M} \frac{1}{y} \int_{0}^{y} s^{t}\left[\frac{G^{\prime}(s)}{G(s)}+r \frac{H^{\prime}(s)}{H(s)}\right] d s d y\right\} .
$$

The boundary terms vanish, so this becomes

$$
-\frac{1}{I} \int_{0}^{M} \frac{1}{y} \int_{0}^{y} s^{t}\left[\frac{G^{\prime}(s)}{G(s)}+r \frac{H^{\prime}(s)}{H(s)}\right] d s d y .
$$

The fact that $\int_{0}^{y} s^{t}\left[\frac{G^{\prime}(s)}{G(s)}+r \frac{H^{\prime}(s)}{H(s)}\right] d s$ is zero at $y=0$ and $y=M$ and the hypothesis about $G H^{r}$ and the consequence for its logarithmic derivative, $\frac{G^{\prime}(s)}{G(s)}+r \frac{H^{\prime}(s)}{H(s)}$, ensure that $\int_{0}^{y} s^{t}\left[\frac{G^{\prime}(s)}{G(s)}+r \frac{H^{\prime}(s)}{H(s)}\right] d s$ is nonnegative for all $y$ in $(0, M)$. This proves the lemma.

Proof of Theorem [2.1. If $X$ has distribution $\operatorname{BIN}(N, p)$ and $c$ is an integer, then $P(X \geq c)=N\left(\begin{array}{c}N-1 \\ c-1\end{array}\right) \int_{0}^{p} x^{c-1}(1-x)^{N-c} d x$. Since $\sum_{j=1}^{n} X_{j}$ has distribution $\operatorname{BIN}(n N, p)$, the theorem will follow if it is shown that

$$
t\left(\begin{array}{c}
t-1 \\
t p-1
\end{array}\right) \int_{0}^{p} x^{t p-1}(1-x)^{t-t p} d x=\frac{\int_{0}^{p} x^{t p-1}(1-x)^{t q} d x}{\int_{0}^{1} x^{t p-1}(1-x)^{t q} d x}
$$

is decreasing in $t$. This will follow from the Lemma with $a=0, b=p, c=1$, $K(x)=x^{p}(1-x)^{q}$, and $M=p^{p} q^{q}$ if it is shown that $G H^{r}$ decreases only on an interval with end point 1 . Implicit differentiation of $K(x)$ gives $G^{\prime}(y)=\frac{G(1-G)}{y(p-G)} \geq 0$ and $H^{\prime}(y)=\frac{H(1-H)}{y(p-H)} \leq 0$. First, it will be shown that $\frac{G^{\prime}(y)}{G(y)}+\frac{H^{\prime}(y)}{H(y)}>0$ by comparing the values of $K(x)$ at the points $p-s$ in $[0, p]$ and $\frac{q p+s(2-q)}{q+2 s}$ in $[p, 1]$. In particular, the ratio

$$
\frac{K(p-s)}{K\left(\frac{q p+s(2-q)}{q+2 s}\right)}=\frac{(p-s)^{p}(q+2 s)}{[q p+(2-q) s]^{p} q^{q}}
$$

is decreasing in $s$ on $(0, p)$. Thus,

$$
\frac{K(p-s)}{K\left(\frac{q p+s(2-q)}{q+2 s}\right)} \leq \frac{K(p-0)}{K\left(\frac{q p+0}{q+0}\right)}=1
$$

and

$$
K(p-s) \leq K\left(\frac{q p+s(2-q)}{q+2 s}\right) .
$$

If $G(y)=p-s$, then $K(G(y))=K(H(y))$ and $K$ decreasing on $[p, 1]$ show that

$$
H(y) \geq \frac{q p+[p-G(y)](2-q)}{q+2[p-G(y)]}=1-\frac{q}{2-\frac{q}{1-G}} .
$$

So, $\frac{q}{2-q /(1-G)} \geq 1-H$ which implies $\frac{q}{1-H} \geq 2-\frac{q}{1-G}$ and

$$
0 \leq \frac{1}{1-\frac{q}{1-G}}+\frac{1}{1-\frac{q}{1-H}}=\frac{1-G}{p-G}+\frac{1-H}{p-H}=\frac{G^{\prime}}{G}+\frac{H^{\prime}}{H} .
$$

Equivalently, this shows that $G(y) H(y)$ is an increasing function of $y$. In particular, $G(y) H(y) \leq G\left(p^{p} q^{q}\right) H\left(p^{p} q^{q}\right)=p^{2}$. It follows that $p^{2}(H-G) \geq G H(H-G)$ and 
$p^{2} H+G^{2} H \geq p^{2} G+G H^{2}$ and $H\left(p^{2}-2 p G+G^{2}\right) \geq G\left(p^{2}-2 p H+H^{2}\right)$ and finally $\frac{H}{(p-H)^{2}} \geq \frac{G}{(p-G)^{2}}$. For any $r>0$ the derivative with respect to $y$ of

$$
y\left[\frac{G^{\prime}}{G}+r \frac{H^{\prime}}{H}\right]=\frac{1-G}{p-G}+r \frac{1-H}{p-H}
$$

is

$$
\frac{q}{y}\left[\frac{G}{(p-G)^{2}} \cdot \frac{1-G}{p-G}+r \frac{H}{(p-H)^{2}} \cdot \frac{1-H}{p-H}\right]
$$

So, if

$$
y\left[\frac{G^{\prime}}{G}+r \frac{H^{\prime}}{H}\right]=\frac{1-G}{p-G}+r \frac{1-H}{p-H} \leq 0,
$$

then $\frac{H}{(p-H)^{2}} \geq \frac{G}{(p-G)^{2}}$ implies that

$$
\frac{q}{y}\left[\frac{G}{(p-G)^{2}} \cdot \frac{1-G}{p-G}+r \frac{H}{(p-H)^{2}} \cdot \frac{1-H}{p-H}\right] \leq 0 .
$$

Thus, if $y\left[\frac{G^{\prime}(y)}{G(y)}+r \frac{H^{\prime}(y)}{H(y)}\right]<0$, for some $y$, then its derivative will also be negative ensuring $\frac{G^{\prime}(y)}{G(y)}+r \frac{H^{\prime}(y)}{H(y)}$ stays negative. This is the result needed to prove Theorem 2.1 .

Proof of Theorem 2.2. Since $\sum_{j=1}^{n} X_{j}$ has a $\operatorname{GAM}(n \alpha)$ distribution, the theorem will follow if it is shown that

$$
\frac{1}{\Gamma(t)} \int_{t}^{\infty} y^{t-1} e^{-y} d y=\frac{\int_{t}^{\infty} y^{t-1} e^{-y} d y}{\int_{0}^{\infty} y^{t-1} e^{-y} d y}
$$

is increasing in $t$. By substituting $x=\frac{y}{t}$, this becomes

$$
\frac{\int_{1}^{\infty} x^{t-1} e^{-t x} d x}{\int_{0}^{\infty} x^{t-1} e^{-t x} d x}
$$

Let $K(x)=x e^{-x}, G(x)$ be the inverse of $K(x)$ on the interval [0,1], and let $H(x)$ be the inverse of $K(x)$ on the interval $[1, \infty)$. The theorem will follow by applying the Lemma with $a=0, b=1, c=\infty$, and $M=\frac{1}{e}$ if the conditions on $G(y)$ and $H(y)$ are verified. Differentiating $K(x)$ implicitly gives $G^{\prime}(y)=\frac{G(y)}{y[1-G(y)]} \geq 0$ and $H^{\prime}(y)=\frac{H(y)}{y[1-H(y)]}<0$. Note that for $t$ in $(0,1)$

$$
\frac{K[1+t]}{K[1-t]}=\frac{1+t}{1-t} e^{-2 t}=\left(1+2 t+2 t^{2}+2 t^{3}+\cdots\right) e^{-2 t}<e^{2 t} e^{-2 t}=1 .
$$

So, for $t$ in $(0,1), K[1-t] \leq K[1+t]$ which implies $K[G] \leq K[2-G]$. Since $K$ is decreasing on $(1, \infty)$ and $K[G]=K[H]$, it follows that $2-G \leq H$ and $2 \leq G+H$. Since $\frac{G^{\prime}}{G}+\frac{H^{\prime}}{H}=\frac{1}{y}\left[\frac{2-G-H}{(1-G)(1-H)}\right]$, it follows that $\frac{G^{\prime}}{G}+\frac{H^{\prime}}{H}>0$ and $G(y) H(y)$ is increasing in $y$. In particular, $G(y) H(y) \leq G(M) H(M)=1$. To apply the Lemma it is sufficient to show that for $r>0$

$$
\frac{G^{\prime}}{G}+r \frac{H^{\prime}}{H}=\frac{1}{y}\left[\frac{1}{(1-G)}+\frac{r}{(1-H)}\right]=\frac{1}{y(H-1)}\left[\frac{H-1}{1-G}-r\right]
$$


remains negative once it becomes negative. This would follow if $\frac{H-1}{1-G}$ were decreasing. Its derivative is

$$
\frac{(1-G) \frac{H}{y(1-H)}+(H-1) \frac{G}{y(1-G)}}{(1-G)^{2}}=\frac{(G H-1)(H-G)}{y(H-1)(1-G)^{3}}
$$

which is always negative since $H \geq 1 \geq G$ and $G H \leq 1$. This proves Theorem 2.2

Theorem 2.3 now follows from the easily established fact that if $X$ has a $\operatorname{POI}(\lambda)$ distribution and $Y$ has a $\operatorname{GAM}(\lambda)$ distribution, then $P(X \geq k)=P(Y<k)$. It also follows from Theorem 2.1 by viewing the Poisson distribution as the limit of binomial distributions.

\section{NUMERICAL EVIDENCE OF MONOTONICITY}

It is very likely that the results of Section 2 will generalize to show that (1.1) exhibits monotonic approach to its limit for values of $m$ other than zero. The following numerical evidence is presented in support of this assertion.

When the $X_{j}$ are binomial random variables, numerical approximations suggest (see Tables 1 and 2) that (1.1) seems to decrease for all values of $p$ in the interval $(0,1)$ when $m$ is small (between $-2 \sqrt{p q}$ and $4 \sqrt{p q}$, for example). On the other hand, when $p=0.4$ and $m=10 \sqrt{p q}$, (1.1) increases to a maximum at about $n=455$ before decreasing for larger $n$. When $p=0.4$ and $m=-10 \sqrt{p q}$, (1.1) appears to oscillate even for large values of $n$.

Similar behavior can be seen when the $X_{j}$ are independent identically distributed random variables with a gamma distribution (see Table 3). For $m=0$, (1.1) strictly increases in $\alpha$ as predicted by Theorem 2.2. For $m<0$, (1.1) decreases for small values of $\alpha$ and increases for larger values. For $m>0$, the behavior is reversed, and (1.1) increases for small values of $\alpha$ and decrease for larger values. The values of $\alpha$ at which the monotonicity changes direction appears to be a smooth function of $m$ (see Table 4). Generalizing Theorem 2.2 would be of great interest, but these changes in direction of the monotonicity for small $\alpha$ make the analysis difficult.

TABle 1. $P\left(X \geq 5 n^{2}+k n\right)$ for $X$ with a $\operatorname{BIN}\left(5 n^{2}, 0.2\right)$ distribution

\begin{tabular}{|l|c|c|c|c|c|c|c|}
\hline$n \backslash k$ & -3 & -2 & -1 & 0 & 1 & 2 & 3 \\
\hline 1 & 1. & 1. & 1. & 0.67232 & 0.26272 & 0.05792 & 0.00672 \\
\hline 2 & 1. & 1. & 0.93082 & 0.58855 & 0.19579 & 0.03214 & 0.00259 \\
\hline 3 & 1. & 0.99677 & 0.90979 & 0.55928 & 0.17411 & 0.02499 & 0.00165 \\
\hline 4 & 0.99997 & 0.99472 & 0.89940 & 0.54452 & 0.16341 & 0.02166 & 0.00125 \\
\hline 5 & 0.99993 & 0.99339 & 0.89317 & 0.53564 & 0.15703 & 0.01975 & 0.00104 \\
\hline 6 & 0.99990 & 0.99245 & 0.88902 & 0.52971 & 0.15279 & 0.01850 & 0.00091 \\
\hline 7 & 0.99987 & 0.99177 & 0.88606 & 0.52547 & 0.14977 & 0.01763 & 0.00082 \\
\hline 8 & 0.99984 & 0.99124 & 0.88384 & 0.52229 & 0.14751 & 0.01698 & 0.00076 \\
\hline 9 & 0.99982 & 0.99082 & 0.88210 & 0.51981 & 0.14575 & 0.01648 & 0.00071 \\
\hline 10 & 0.99980 & 0.99049 & 0.88072 & 0.51783 & 0.14435 & 0.01609 & 0.00068 \\
\hline 11 & 0.99979 & 0.99021 & 0.87958 & 0.51621 & 0.14320 & 0.01577 & 0.00065 \\
\hline 12 & 0.99977 & 0.98998 & 0.87864 & 0.51486 & 0.14225 & 0.01550 & 0.00062 \\
\hline
\end{tabular}


Table 2. $P\left(X \geq 5 n^{2}+k n\right)$ for $X$ with a $\operatorname{BIN}\left(5 n^{2}, 0.6\right)$ distribution

\begin{tabular}{|l|c|c|c|c|c|c|c|}
\hline$n \backslash k$ & -3 & -2 & -1 & 0 & 1 & 2 & 3 \\
\hline 1 & 1. & 0.98976 & 0.91296 & 0.68256 & 0.33696 & 0.07776 & \\
\hline 2 & 0.99838 & 0.97897 & 0.87247 & 0.59559 & 0.25001 & 0.05095 & 0.00361 \\
\hline 3 & 0.99785 & 0.97497 & 0.85635 & 0.56429 & 0.22490 & 0.04463 & 0.00364 \\
\hline 4 & 0.99760 & 0.97287 & 0.84772 & 0.54836 & 0.21311 & 0.04174 & 0.00356 \\
\hline 5 & 0.99745 & 0.97157 & 0.84235 & 0.53874 & 0.20627 & 0.04009 & 0.00349 \\
\hline 6 & 0.99735 & 0.97069 & 0.83868 & 0.53231 & 0.20181 & 0.03901 & 0.00343 \\
\hline 7 & 0.99729 & 0.97005 & 0.83603 & 0.52771 & 0.19867 & 0.03826 & 0.00339 \\
\hline 8 & 0.99724 & 0.96957 & 0.83401 & 0.52425 & 0.19634 & 0.03770 & 0.00335 \\
\hline 9 & 0.99720 & 0.96919 & 0.83243 & 0.52156 & 0.19455 & 0.03727 & 0.00333 \\
\hline 10 & 0.99717 & 0.96888 & 0.83116 & 0.51941 & 0.19312 & 0.03692 & 0.00330 \\
\hline 11 & 0.99714 & 0.96863 & 0.83011 & 0.51764 & 0.19196 & 0.03665 & 0.00329 \\
\hline 12 & 0.99712 & 0.96842 & 0.82923 & 0.51617 & 0.19099 & 0.03642 & 0.00327 \\
\hline
\end{tabular}

TABle 3. $P(X \geq \alpha+m \sqrt{\alpha})$ for $X$ with a $\operatorname{GAM}(\alpha)$ distribution

\begin{tabular}{|l|c|c|c|c|c|c|c|c|c|}
\hline$\alpha \backslash m$ & -1.00 & -0.75 & -0.50 & -0.25 & 0.00 & 0.25 & 0.50 & 0.75 & 1.00 \\
\hline 2 & 0.88275 & 0.75806 & 0.62934 & 0.51005 & 0.40600 & 0.31869 & 0.24737 & 0.19026 & 0.14523 \\
\hline 4 & 0.85712 & 0.75757 & 0.64723 & 0.53663 & 0.43347 & 0.34229 & 0.26502 & 0.20169 & 0.15120 \\
\hline 6 & 0.85086 & 0.75918 & 0.65532 & 0.54827 & 0.44568 & 0.35291 & 0.27293 & 0.20661 & 0.15343 \\
\hline 8 & 0.84810 & 0.76050 & 0.66017 & 0.55515 & 0.45296 & 0.35929 & 0.27766 & 0.20949 & 0.15462 \\
\hline 10 & 0.84656 & 0.76154 & 0.66348 & 0.55983 & 0.45793 & 0.36366 & 0.28089 & 0.21142 & 0.15535 \\
\hline 20 & 0.84374 & 0.76450 & 0.67170 & 0.57136 & 0.47025 & 0.37457 & 0.28895 & 0.21611 & 0.15691 \\
\hline 40 & 0.84248 & 0.76687 & 0.67750 & 0.57944 & 0.47897 & 0.38233 & 0.29466 & 0.21932 & 0.15774 \\
\hline 60 & 0.84208 & 0.76799 & 0.68007 & 0.58300 & 0.48283 & 0.38579 & 0.29720 & 0.22071 & 0.15803 \\
\hline 80 & 0.84189 & 0.76868 & 0.68160 & 0.58511 & 0.48513 & 0.38786 & 0.29872 & 0.22153 & 0.15818 \\
\hline 100 & 0.84177 & 0.76915 & 0.68264 & 0.58655 & 0.48670 & 0.38927 & 0.29975 & 0.22208 & 0.15827 \\
\hline 200 & 0.84155 & 0.77035 & 0.68522 & 0.59013 & 0.49059 & 0.39278 & 0.30232 & 0.22344 & 0.15846 \\
\hline 400 & 0.84144 & 0.77121 & 0.68705 & 0.59264 & 0.49335 & 0.39526 & 0.30414 & 0.22439 & 0.15855 \\
\hline 600 & 0.84141 & 0.77160 & 0.68786 & 0.59376 & 0.49457 & 0.39637 & 0.30494 & 0.22480 & 0.15858 \\
\hline 800 & 0.84139 & 0.77184 & 0.68834 & 0.59442 & 0.49529 & 0.39702 & 0.30542 & 0.22505 & 0.15860 \\
\hline 1000 & 0.84138 & 0.77200 & 0.68867 & 0.59487 & 0.49579 & 0.39747 & 0.30575 & 0.22522 & 0.15861 \\
\hline 10000 & 0.84134 & 0.77293 & 0.69058 & 0.59749 & 0.49867 & 0.40008 & 0.30765 & 0.22618 & 0.15865 \\
\hline
\end{tabular}

TABLE 4. Values of $\alpha$ where $P(X \geq \alpha+m \sqrt{\alpha})$ changes direction, $X$ is $\operatorname{GAM}(\alpha)$

\begin{tabular}{|c|l|l|l|l|l|l|l|l|l|}
\hline$m$ & -1.00 & -0.95 & -0.90 & 0.85 & -0.80 & -0.75 & 0.70 & -0.65 & -0.60 \\
\hline$\alpha$ & 1956.69 & 93.188 & 21.700 & 8.990 & 4.721 & 2.828 & 1.840 & 1.265 & 0.902 \\
\hline \hline$m$ & 1.00 & 1.05 & 1.10 & 1.15 & 1.20 & 1.25 & 1.30 & 1.35 & 1.40 \\
$\alpha$ & 13,812 & 107.16 & 28.654 & 13.588 & 8.133 & 5.522 & 4.056 & 3.144 & 2.531 \\
\hline
\end{tabular}


These simple cases of monotonic convergence are only special cases of a more general phenomenon, the monotonicity of conditional probabilities. For example, let $X_{1}, X_{2}$, and $X_{3}$ be independent random variables with a $\operatorname{BIN}(N, p)$ distribution, $\mu=N p$, and $\sigma=\sqrt{N p q}$. Discussions by Chover [1994, 1996] require an estimate for

$$
\begin{aligned}
& P\left(X_{1}+X_{2} \geq \mu+n \sigma \mid X_{1}+X_{3} \geq \mu+m \sigma\right) \\
& =\frac{P\left(X_{1}+X_{2} \geq \mu+n \sigma \& X_{1}+X_{3} \geq \mu+m \sigma\right)}{P\left(X_{1}+X_{3} \geq \mu+m \sigma\right)} .
\end{aligned}
$$

In particular, Chover makes this estimate for $p=0.2, n=1, m=1.5$, and $N$ large. A normal approximation of 0.4852 is used. How accurate is this approximation? The numerator, the denominator, and the fraction itself all appear to be monotone in $N$ (see Table 5) making the accuracy of the estimate easy to evaluate. Similar behavior was observed for a wide range of choices of $p, n$, and $m$.

If the $X_{1}, X_{2}$, and $X_{3}$ in the above conditional probability have a $\operatorname{GAM}(\alpha)$ distribution rather than a $\operatorname{BIN}(N, p)$ distribution, the same monotonicity phenomenon is observed with $\mu=\alpha$ and $\sigma=\sqrt{\alpha}$ (see Table [6).

TABLE 5. $P\left(X_{1}+X_{2} \geq \mu+\sigma \mid X_{1}+X_{3} \geq \mu+1.5 \sigma\right), X_{j}$ is $\operatorname{BIN}(N, 0.2)$

\begin{tabular}{|r|c|c|c||c|c|c|c|}
\hline$N$ & numerator & denominator & ratio & $N$ & numerator & denominator & ratio \\
\hline 50 & 0.0454 & 0.0874 & 0.520 & 1250 & 0.0348 & 0.0708 & 0.492 \\
\hline 200 & 0.0387 & 0.0770 & 0.502 & 1800 & 0.0344 & 0.0701 & 0.491 \\
\hline 450 & 0.0365 & 0.0735 & 0.496 & 2450 & 0.0341 & 0.0697 & 0.490 \\
\hline 800 & 0.0355 & 0.0718 & 0.494 & 3200 & 0.0339 & 0.0693 & 0.489 \\
\hline
\end{tabular}

TABle 6. $P\left(X_{1}+X_{2} \geq \mu+\sigma \mid X_{1}+X_{3} \geq \mu+1.5 \sigma\right), X_{j}$ is $\operatorname{GAM}(\alpha)$

\begin{tabular}{|r|c|c|c||c|c|c|c|}
\hline$\alpha$ & numerator & denominator & ratio & $\alpha$ & numerator & denominator & ratio \\
\hline 50 & 0.0350 & 0.0716 & 0.4895 & 400 & 0.0334 & 0.0686 & 0.4886 \\
\hline 100 & 0.0343 & 0.0703 & 0.4882 & 800 & 0.0331 & 0.0681 & 0.4862 \\
\hline 150 & 0.0339 & 0.0697 & 0.4876 & 1200 & 0.032995 & 0.067882 & 0.4860 \\
\hline 200 & 0.0337 & 0.0693 & 0.4873 & 1400 & 0.032953 & 0.067804 & 0.4859 \\
\hline
\end{tabular}

\section{ACKNOWLEDGMENTS}

The author would like to thank Joshua Chover and Richard Askey who offered helpful suggestions along the way to completing this research.

\section{REFERENCES}

[1994] J. Chover, Recall via Transient Neuronal Firing, Neural Networks, 7 (1994), 233-250.

[1996] J. Chover, Neural Correlation via Random Connections, Neural Computation 8 \#8, (1996), $1711-1729$.

[1968] Kumar Jogdeo and S. M. Samuels, Monotone Convergence of Binomial Probabilities and a Generalization of Ramanujan's Equation, Ann. Math. Stat. 39 (1968), 1191-1195.

Department of Mathematical and Computer Sciences, University of Wisconsin, WhiteWater, 800 West Main Street, Whitewater, Wisconsin 53190-1790

E-mail address: kanej@mail.uww.edu 